\title{
Anabases
}

ANABASES Traditions et réceptions de l'Antiquité

$21 \mid 2015$

Varia

\section{Anne ROLET (éd.), Allégorie et symbole : voies de dissidence ? De l'Antiquité à la Renaissance}

\section{Cyrielle Landrea}

\section{OpenEdition}

\section{Journals}

Édition électronique

URL : http://journals.openedition.org/anabases/5365

DOI : $10.4000 /$ anabases.5365

ISSN : 2256-9421

\section{Éditeur}

E.R.A.S.M.E.

\section{Édition imprimée}

Date de publication : 1 avril 2015

Pagination : 287-288

ISSN : 1774-4296

\section{Référence électronique}

Cyrielle Landrea, «Anne Rolet (éd.), Allégorie et symbole : voies de dissidence ? De l'Antiquité à la

Renaissance ", Anabases [En ligne], 21 | 2015, mis en ligne le 01 avril 2015, consulté le 22 septembre

2020. URL : http://journals.openedition.org/anabases/5365 ; DOI : https://doi.org/10.4000/anabases 5365

Ce document a été généré automatiquement le 22 septembre 2020

(c) Anabases 


\title{
Anne ROLET (éd.), Allégorie et symbole : voies de dissidence ? De l'Antiquité à la Renaissance
}

\author{
Cyrielle Landrea
}

\section{RÉFÉRENCE}

Anne RoLEt (éd.), Allégorie et symbole : voies de dissidence ? De l'Antiquité à la Renaissance, Rennes, Presses Universitaires de Rennes, 2012, 597 p.

24 euros / ISBN 9782753519824.

1 Issu d'un colloque international en 2009, ce volume dense a pour volonté de se situer au point de convergence entre d'un côté l'allégorie et le symbole - voire le mythe - et de l'autre les dissidences. L'approche est résolument diachronique, puisque l'ouvrage couvre une période allant de l'Antiquité à la Renaissance et même jusqu'au XIX $\mathrm{s}$.

2 L'introduction, bien menée et d'une grande érudition, replace bien le débat, puis définit clairement les concepts d'allégorie, de symbole et de dissidence à travers les âges, y compris d'un point de vue étymologique. L'approche sur le temps long n'était pas un prétexte, même s'il existe une distinction entre les grandes périodes historiques, rendue effectivement nécessaire, car l'allégorie et le symbole ne sont pas perçus de la même façon.

3 L'entreprise était vaste et dans la perspective du colloque, des domaines de réflexion cohérents et complémentaires ont été privilégiés autour de plusieurs grands thèmes fédérateurs. Dans la première partie intitulée : « L'allégorie mode de construction des oppositions politiques, intellectuelles et artistiques dans l'Antiquité », les articles ont pour but d'explorer le devenir de l'allégorie et de quelques usages idéologiques liés à la dissidence dans l'Antiquité. S. Kefallonitis démontre le refus originel des historiens grecs d'associer l'allégorie à leur récit, puis sa progressive utilisation à l'époque romaine (uia l'allegoria in factis) pour mieux rendre compte des éléments exemplaires et 
universels. Le parcours de Q. Lutatius Catulus (cos. 102 av. J.-C.) - anticonformiste notoire - est mis en perspective par G. Sauron, tandis que J. Fabre-Serris montre comment Ovide a prôné l'élégie et l'érotisme au milieu du moralisme ambiant, sans pour autant être un dissident. Enfin la récupération de l'allégorie et du symbole par l'opposition politique au I ${ }^{\mathrm{er}} \mathrm{s}$. ap. J.-C. est appréciée par C. Badel. Cependant la portée de cette opposition est brouillée par une tradition culturelle commune, la bataille des allégories et la récupération impériale.

4 La perspective est élargie dans la deuxième partie (" Lectures philosophiques de l'allégorie antique : la contestation de la tradition »). A.Gigandet insiste sur l'opposition de Lucrèce à l'allégorèse classique des mythes, tandis que B. Pouderon examine les débats autour du vocabulaire de l'allégorie chez Héraclite Pontique. Enfin la philosophie (Sénèque, Apulée et Marc Aurèle) a dû affronter des querelles qui portaient sur la pauvreté dans les allégories romaines impériale (J. Dross).

5 Dans la troisième partie (« Le symbole et l'allégorie dans la confrontation entre références païennes et culture judéo-chrétienne »), les communications étudient les formes de résistance à l'assimilation religieuse et les modalités d'attraction d'une religion à l'autre. Les propos portent d'abord sur le recours à des signes discrets de reconnaissance à l'époque proto-chrétienne lorsqu'il était dangereux de s'afficher comme chrétien (I. Ramelli). Puis la croyance dans la résurrection du corps martyrisé chez les Juifs fait émerger une symbolique singulière (M.-F. Baslez). Le païen Procope fait des allusions aux mystères eucharistiques (E. Amato), alors que le chrétien Ennode de Pavie utilise des allégories païennes (V. Zarini).

6 La quatrième partie nous fait entrer dans l'ère médiévale (" Nouvelles sensibilités spirituelles et mutations du langage allégorique à l'époque médiévale »). Afin de mettre en avant les continuités et les ruptures entre l'Antiquité tardive et la Renaissance, plusieurs études sont proposées à commencer par les mouvements philosophiques et spirituels $\left(\mathrm{XI}^{\mathrm{e}}-\mathrm{XIV}^{\mathrm{e}} \mathrm{s}\right.$.) qui remettent en cause les allégories religieuses (S. Piron) ou celle sur la traduction de l'Ovide moralisé en langue romane, qui lui donne un sens plus chrétien (M. Possamai-Pérez). Ensuite la propagande révolutionnaire de Cola di Rienzo a su utiliser de façon originale les personnifications allégoriques traditionnelles de Rome (J.C. d'Amico).

7 Les usages différents et pléthoriques de l'allégorie à la Renaissance dans le cadre des oppositions religieuses sont au cœur de la cinquième partie (" Allégorie plastique, allégorie littéraire dans les controverses religieuses $d u \mathrm{xvI}^{\mathrm{e}} \mathrm{s}$. »). Certaines figures (comme Brutus) ont été utilisées à l'époque de la Pléiade et d'Etienne Pasquier pour dénigrer les protestants (E. Karagiannis), tandis que des personnifications peintes par Vasari visent à soutenir un idéal religieux renouvelé (A. Fenech Kroke). La nomination des mois fut aussi au cœur de querelles entre calvinistes et catholiques (E. Leutrat).

8 "L'allégorie à la Renaissance, arme des dissidences " est la sixième partie qui s'intéresse aux symboles antiques dans la célébration du tyrannicide (S. Rolet), aux pouvoirs subversifs de l'humour dans la peinture allégorique vénitienne (G. Cassegrain), au rôle de l'allégorie dans les allusions homosexuelles chez Ronsard (P. Ford), et aux allégories pour dire et masquer de nouvelles conceptions scientifiques au XVII ${ }^{\mathrm{e}}$ s. (A. Guiderdoni-Bruslé).

9 Enfin la dernière partie (« Dissidence et circulation géographique de l'allégorie à la Renaissance ») montre les échanges entre les cours de Ferrare et de Turin (R. Gorris 
Camos), le langage symbolique à Bâle pour attirer Érasme (V. Sebastiani) et les métamorphoses de l'âne-Pégase (O. Pot). Les allégories dans la musique de Liszt servent d'épilogue (P. Maréchaux).

10 Un des intérêts de l'ouvrage réside dans la complémentarité des approches mêlant l'épistémologie, l'histoire, l'histoire de l'art, mais aussi les approches idéologiques, littéraires, musicologiques, philosophiques et religieuses. Notons aussi la présence bienvenue d'un index pour mettre en relation les articles.

\section{AUTEURS}

\section{CYRIELLE LANDREA}

Paris, ANHIMA (UMR 8210)

cyrielle.landrea@orange.fr 